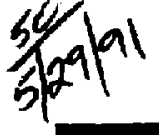

PREPARED FOR THE U.S. DEPARTMENT OF ENERGY, UNDER CONTRACT DE-AC02-76-CHO-3073

PPPL-2750

PPPL-2750

UC-420,427

\section{THE MODIFICATION OF TURBULENT TRANSPORT BY ORBIT AVERAGING}

\section{BY}

H.E. MYNICK AND S.J. ZWEBEN

May 1991

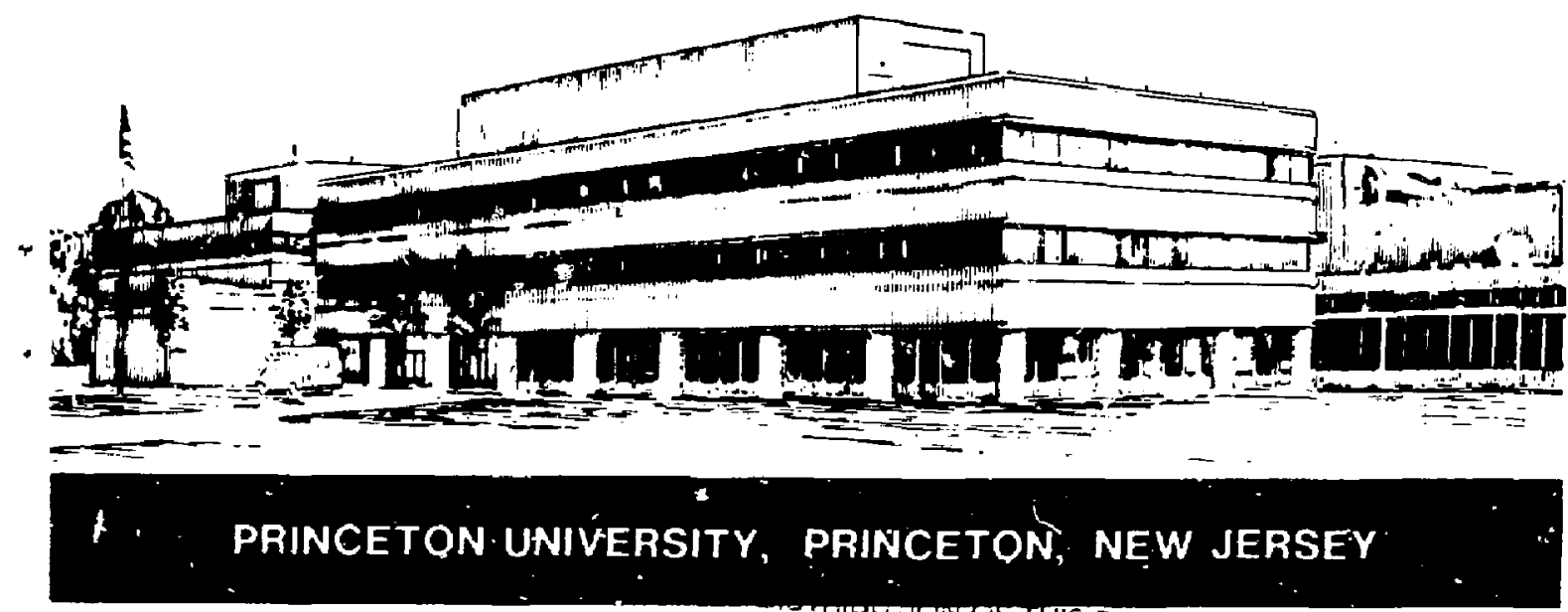

$t$ 


\section{MOTICE}

This report was prepared as an account of work sponsored by an agency of the United States Government. Neither the United States Government nor any agency thereof, nor any of their employees, makes any warranty, express or implied, or assumes any legal liability or responsibility for tite accurac:" completeness, or usefulness of any information, apparatus, product, or process disclosed, or represents that its use would not infringe privately owned rights. Reference herein to any specific commercial produce, process, or service by trade name, trademark, manufacturer, or otherwise. does not necessarily constitute or imply its endorsement, recommendation, or favoring by the United States Guvernment or any agency thereot. The views and opinions of authors expresced herein do not necessarily state or reflect those of the United States Government or any agency thereof.

\section{NOTICE}

This report has been reproduced directly from the best avaiiable copy.

Available to DOE and DOE contractors from the:

Office of Scientific and Technical Information

P.O. Box 62

Oak Ridge, TN 37831;

Prices available irom (615) 576-8401.

Available to the public trom the:

National Technical Information Service

U.S. Department of Commerce 5285 Port Royal Road

Springfield, Virginia 22161

$703-487-4650$ 


\title{
The Modification of Turbulent Transport by Orbit Averaging
}

\author{
H.E. Mynick and S.J. Zweben \\ Plasma Physics Laboratory, Princeton University \\ P.O. Box 451 \\ Princeton, New Jersey 08543-0451, U.S.A.
}

\begin{abstract}
The effect on plasma turbulence of orbit averaging by thermal jons is considered, and illustrated for two modes of potential importance for tokamaks. The effect can reduce the ion response below that in earlier treatments, modifying the predicted mode growth rate, which in turn modifies the turbulent transport. For both modes, the effect modifies earlier transport expressions wit'i a 'neoclassical factor, 'which makes the scalings of the resultant transport coefficients with plasma current and magnetic field closer to those found experimentally. Addicionally, for the trapped electron mode, this mechanisn provides a potential explanation of the observed more favorable scaling of $\chi_{i}$ with $T_{i}$ in supershots than in L-modes.
\end{abstract}

For some time, it has been appreciated theoretically ${ }^{1}$ that the net effect of field fuctuations on particles with appreciable gyroradii $\rho_{g}$ and drift excursions $\rho_{b}$ can be substantially different from (usually, smaller than) their effect in the $\rho_{b, s} \rightarrow 0$ limit, due to an averaging over the fluctuation phase 
which a particle with finite Larmor radius and finite drifts performs in the course of a gyroperiod $\tau_{g}$ or bounce (or transit) period $\tau_{b}$. This reduction in interaction strength causes a corresponding reduction in the transport levels induced by a given spectrum of fluctuations. This theoretical expectation is supported by experimental observalires of transport rates of energetic electrons ${ }^{3}$ and ions, ${ }^{4,5}$ as well as $b$...hical simulations. ${ }^{6}$

Here, we consider a complementary effect less widely appreciated, namely: that : r only does orbit averaging diminish the extent to which a specified spectrum of fluctuations affects particles (as manifested by the transport coefficients $D$ or $\chi)^{1,2}$ but also it diminishes the extent to which particles affect fluctuations (as manifested by the lineas susceptibility $K$ ), and hence modifies the stability of modes which cause turbulent transport. We apply this basic idea to two illustrative modes which may be important in causing anomolous transport in tokamaks, the trapped electron mode (TEM) ${ }^{7}$ and the ion temperature gradient (ITG) mode, ${ }^{8}$ and obtain estimates for the modification by orbit averaging of tokamak turbulent transport coefficients. The averaging due to finite $p_{b}$ introduces an extra $q$-dependence into the transport coefficients, similar to the one encountered in going from classical to neoclassical transport, which brings the resultant scalings with current $I_{p}$ and magnetic field $B$ into closer agreement with those observed experimentally. Moreover, the modified expressions for the TEM predict an important effect observed on TFTR, 9,10 namely, the decrease in $\chi_{i}$ with increased $T_{i}$ for supershots, for which $\left.T \equiv T_{e} / T_{i} \ll 1\right)$, in contrast to the usual increase in $\chi_{i}$ with increased $T_{i}$ in L-mode plasmas (where $\tau \sim 1$ ) (see Fig.1, from $10)$.

The formal theory permitting study of both types of orbit-averaging 
effects was developed by Kaufman, ${ }^{11}$ who used an action-angle framework to develop expressions for both the quatilinear diffusion tensor $\mathbf{D}$ as well as the linear susceptibility $K$, valid for a range of unperturbed magnetic geometries (including slabs and tokamaks), and for artitrary mode structure. Application of the formal expression for $\mathbf{D}$ to tokamak transport problems was made in Refs. 1,2,12-14. The structure of $K$, of principal interest here, is quite similar. Both are given by a sum over all harmonics $I \equiv\left(l_{g}, l_{b}, l_{\zeta}\right)$ of the gyro-, bounce-, and tocoidal precession frequencies $\left(\Omega_{g}, \Omega_{b}, \Omega_{\zeta}\right)$, with an 'orbit-averaging factor' $G_{\mid}^{2}$, whose effect is central here, weighting the summand. Axisymmetry requires that $l_{\zeta}=n_{1}$ and one may take only $l_{s}=0$ components for the low frequency modes considered here. As usual, we parametrize the tokamak by the radial, poloidal, and toroidal coordinates $(r, \theta, \varsigma)$. Simplifying somewhat for purposes of brevity and physical clarity, we model the mode structure as locally a plane wave with wave vector $k=r k_{r}+\hat{\theta} m / r+\bar{\zeta} n / R$. Then for species $s, g_{s} \equiv k^{2} \lambda_{j}^{2} K_{s}$ is approximately given by ( $\lambda_{g}$ is the Debye length)

$$
g_{s}(k, \omega)=1-\sum_{l}\left(J_{0}^{2}\left(z_{g}\right) J_{l}^{2}\left(z_{b}\right) \frac{\omega-\omega_{s}^{\prime}}{\omega-\sigma k_{\|\|}} \overline{\bar{v}_{\|}-(1-\sigma) \omega_{d_{s}}-1 \Omega_{b}+i \nu_{s}} j\right.
$$

Here, $l \equiv l_{b}-\sigma m$, with trapping state index $\sigma=0$ for trapped particles, and $\sigma=1$ for passing particles, $\langle\ldots\rangle \equiv \int d v\left(f_{0} / n_{0}\right) \ldots$ is the velocity space average over the unperturbed distribution function fo (species label suppressed ), $\omega_{*}^{\prime} \equiv \omega_{*}\left[1+\eta\left(u^{2}-3\right) / 2\right]$, with $\omega_{*} \equiv-k_{v} c T /\left(e B L_{n}\right)$ the diamagnetic drift frequency, $\eta \equiv d \ln T / d \ln n, u \equiv v / v$, the particle velocity, normalized to the thermal speed $v_{s}, k_{y} \equiv \hat{b} \times \hat{r} \cdot \mathbf{k}$, and $L_{n}^{-1} \equiv-\partial \ln n_{0} / \partial r$. $\bar{v}_{\|}$is the bounce-averaged parallel velocity, $\omega_{\phi}=n \Omega_{\zeta}(\sigma=0)$ is the toroidal 
drift frequency, and $\nu_{f} \simeq: / \epsilon$ is the effective collision frequency, with $\nu$ the 90-degree scattering frequency. For the chosen model of mode structure, $G_{1}^{2}$ is given by the product of the squares of Bessel functions $J^{2}$ appearing in (1), with one $\left(J_{g}^{2} \equiv J_{0}^{2}\left(z_{g}\right)\right)$ due to gyro-orbit-averaging, with argument $z_{g}=k_{\perp} \rho_{g}$, and the other $\left(J_{b}^{2} \equiv J_{f}^{2}\left(z_{b}\right)\right)$ with argument $z_{b}$, due to averaging over the drift orbit. The full expression ${ }^{2,14}$ for $z$ is slightly more complicated than that for $z_{g}$, but the essential physics is captured by approximating it as $z_{b} \simeq k_{\perp} \rho_{b}$. The principal modifying effect on the dispersion relation held in Eq. (1) arises from the finite-drift factor $J_{b}^{2}$ for ions.

For electrons, the treatment is standard. One has $z_{g, b} \rightarrow 0$, so that $G^{2} \rightarrow \delta(l)$, as usual. Making the standard assumption $\xi_{e} \equiv \omega / k_{\eta} v_{e} \ll 1$, the nonadiabatic portion of the electron response is dominated by trapped particles: $g_{e}=1-i \alpha_{e}$, where $i \alpha_{e} \equiv \epsilon^{1 / 2}\left(\left(\omega-\omega_{e}^{\prime}\right) /\left(\omega-\omega_{d e}+i \nu_{f e}\right)\right)_{t}$, with $\langle\ldots\rangle$ : the average over the trapped portion of the distribution.

For ions, if one makes the conventional assumption that $\omega / \Omega_{6} \gg 1$, then over the l-range $\Delta r \sim z_{b}$ over which $J_{l}^{2}$ in Eq. (1) is appreciable, the denominator there does not change greatly, so that one can perform the summation, using the identity $\sum_{l} J_{l}^{2}=1$, which eliminates the $J_{b}^{2}$ factor, leaving only the usual factor $J_{g}^{2}$. In actuality, however, for the modes considered here, one has $\omega / \Omega_{b} \gtrsim 1$, so that only a small number of bounce harmonics, having $\omega /|/| \Omega_{b} \gtrsim 1$, contribute appreciably to the sum, ${ }^{15}$ and the effect of $J_{b}^{2}$ survives. For simplicity, we assume that only the $l=0$ term satisfies this for passing particles, which is often the case, and the sesultant scaling is similar to the more general situation where a few low-l harmonics must be kept. When this is the case, since $\Omega_{b}(\sigma=0) / \Omega_{b}(\sigma=1) \simeq \epsilon^{1 / 2}$, a few low-l harmonics for trapped particles contribute, reducing the drift averaging effect 
for $\sigma=0$. Thus, here we neglect the drift averaging for $\sigma=0$, noting that at somewhat lower frequencies $\left(\omega / \Omega_{b}(\sigma=0) \sim 1\right)$ it will be present for $\sigma=0$ as well as for $\sigma=1$, somewhat strengthening the overall effect. For the single $\sigma=1$ term we assume that the usual condition $\xi \equiv \xi_{i} \equiv \omega / k_{\|} v_{i}>1$ is (marginally) satisfied, and neglect $\omega_{d i}$ and $\nu_{f_{i}}$ in (1). Expanding expression (1) to third order in $\xi^{-1}$, one may evaluate $g_{i}$ in terms of velocity space moments of $G^{2}$, such as $\Lambda_{0} \equiv\left\langle J_{g}^{2} J_{b}^{2}\right\rangle$ and $\Lambda_{2} \equiv\left\{\left(u_{\perp}^{2} / 2\right) J_{g}^{2} J_{b}^{2}\right\rangle$. In the driftless (slab) limit $z_{b} \rightarrow 0$ (with $z_{g}$ fixed), $J_{b}^{2} \rightarrow 1$, the parallel and perpendicular integrations in Eq. (1) decouple, and can be exactly evaluated, in terms of $\Lambda_{0}^{(0)}\left(b_{g}\right) \equiv\left\langle J_{g}^{2}\right)=I_{0}\left(b_{g}\right) e^{-b_{g}}, \Lambda_{2}^{(0)}\left(g_{g}\right) \equiv\left\langle\left(u_{\perp}^{2} / 2\right) J_{g}^{2}\right\rangle=\left(I_{0}+b_{g} I_{0}^{\prime}\right) e^{-b_{g}, \text { and }}$ the plasma dispersion function $\left\langle\left(u_{\|}-\xi\right)^{-1}\right\rangle$. Here, $I_{0}(b)$ is the modified Bessel function of the first kind, and $b_{g} \equiv k_{1}^{2} \rho_{g i}^{2} \equiv k_{\perp}^{2} v_{i}^{2} / \Omega_{g i}^{2}$.

While $\rho_{g}$ and $\rho_{b}$ have the same energy scaling $\left(\propto E^{1 / 2} \propto v\right)$, the pitchangle dependence of $\rho_{b}$ is more complicated than that for $\rho_{g}$, coupling the parallel and perpendicular integrations. Therefore, to capture the essential effect of firite $z_{b}$, we adopt an approximate form for $\Lambda_{0}, \Lambda_{0}\left(b_{g}, b_{b}\right) \simeq(1+$ $\left.b_{c}\right)^{-1} \equiv\left(1+k_{\perp}^{2} \rho_{c}^{2}\right)^{-1}$ and analogous approximations for the higher moments such as $\Lambda_{2}$. Hère, $b_{c} \equiv b_{g}+b_{b}$, with $b_{b} \equiv k_{1}^{2} \rho_{b i}^{2} \simeq k_{1}^{2}\left(q \rho_{g i}\right)^{2}$ the bounceanalogue of $b_{g}$. This choice properly models the slab beltavior $\Lambda_{0}\left(b_{g} \ll\right.$ $\left.1, b_{b}=0\right)=\Lambda_{0}^{(0)}\left(b_{g}\right) \simeq 1-b_{g}$, with a similar linear behavior for $b_{g}=0, b_{b} \ll$ 1 , and the limit $\Lambda_{0}\left(b_{g}, b_{b} \rightarrow \infty\right) \rightarrow 0$. Our treatrnent of $g_{b}$ will thus be to evaluate it exactly in the driftless limit $b_{b} \rightarrow 0$ (but $b_{g}$ arbitrary), and then to replace the $\Lambda^{(0)}$ s which appear by the corresponding $\Lambda s$, which incorporate the effect of of finite $b_{b}$ in the approximate fashion just indicated.

Thus, expanding the dispersion function to third order in $\xi^{-1}$, quasineu- 
trality yields the dispersion equation

$$
\begin{aligned}
0 & =g_{e}+r g_{i} \\
& =(1-i \alpha)-\frac{\omega_{* e}}{\omega}+\tau\left(1-\Lambda_{0}\right)\left(1+\frac{\omega_{* \varepsilon}}{\omega} K_{3}\right)-\frac{k_{\|}^{2} c_{3}^{2}}{\omega^{2}} \Lambda_{0}\left(1+\frac{\omega_{* \varepsilon}}{\omega} K_{4}\right),
\end{aligned}
$$

where $c_{j}^{2} \equiv \tau v_{i}^{2}$ as usual, use has been made of $\tau \omega_{n i}=-\omega_{n e}$, and $\alpha \equiv$ $\alpha_{e}+\tau \alpha_{i}$, with $\alpha_{e}$ and $\alpha_{i} \simeq\left(\omega_{* i} / \omega-1\right) \Lambda_{0} \sqrt{\pi / 2} \xi \exp \left(-\xi^{2} / 2\right)$ containing the resonant contributions from electrons and ions. $\alpha_{i}$ is usually neglected under the $\xi \gg 1$ assumption. Eq. (2) is written in a form which transparently reduces to the small- $b_{g}$ limit usually considered. The first term is $g_{e}-i r \alpha_{i}$, and the remaining three are the test of $\tau g_{i}$. In the third and foutth terms, $K_{3} \equiv\left[1+\eta_{i}\left(-\delta \Lambda_{2} /\left(1-\Lambda_{0}\right)\right)\right] / \tau$ and $K_{4} \equiv\left[1+\eta_{i}\left(\Lambda_{2} / \Lambda_{0}\right)\right] / \tau$, where $\delta \Lambda_{2} \equiv$ $\Lambda_{2}-\Lambda_{0}$. For $b_{s} \ll 1, K_{3}, K_{4} \rightarrow K \equiv\left(1+\eta_{i}\right) / \tau$, and $\left(1-\Lambda_{0}\right), \delta \Lambda_{2} \rightarrow b_{9}$, and one recovers the standard dispersion relation. ${ }^{8}$

Eq. (2) contains both the TEM and the (slablike branch) of the ITG mode, both being drift waves, destabilized by different driving inechanisms. ${ }^{16}$ For the simplest version of the TEM, one sets $\eta_{i}=0=k_{\|}$, resulting in a mode with frequency $\omega=\omega_{r}+i \gamma$, with real and imaginary parts

$$
\omega_{r}=\omega_{m e} \Lambda_{0} /\left[1+\tau\left(1-\Lambda_{0}\right)\right], \gamma=\alpha \omega_{-e} \Lambda_{0} /\left[1+\tau\left(1-\Lambda_{0}\right)\right]^{2}
$$

For the fluidlike limit ( $r>1, b_{g} \ll 1$ ) commonly considered, the denominator in $\gamma$ governs the falloff of $\gamma$ with $k_{\perp}$. Taking $b_{b}=0$ and $\Lambda_{0} \simeq 1-b_{g}$, as is conventional, one has $\gamma \simeq \alpha \omega_{-e} /\left(1+b_{s}^{2}\right)^{2}$, where $b_{s} \equiv \tau b_{g} \equiv k_{1}^{2} \rho_{s}^{2}$. (Fig.2.) Thus, the range in $k_{\perp}$ of $\gamma$ (and accordingly, one expects, of the spectrum) is given by $\Delta k_{1} \simeq \rho_{s}^{-1}$ which scales as $T_{c}^{-1 / 2}$ :

$$
\gamma\left(\tau \geq 1, b_{b}=0\right) \propto k_{y} /\left(1+k_{\perp}^{2} p_{s}^{2}\right)^{2} .
$$


In the same fluidlike limit, but with $b_{b}$ finite, $\Lambda_{0} \simeq 1-b_{c}$, so that $b_{s}$ in the above formula should be approximately replaced by $b_{t} \equiv \tau b_{c} \equiv k_{\perp}^{2} p_{t}^{2}$. $\Delta k_{\perp} \simeq \rho_{t}^{-1}$ thus still scales as $T_{e}^{-1 / 2}$.

However, for $\tau<1$, as is the case in supershots, $\Delta k_{\perp}$ is instead determined by the factor $\Lambda_{0}$ in the numerator of $\gamma$, whose falloff depends upon $T_{i}$, and is more rapid for $r<1$ (Fig.2):

$$
\because(r<1) \propto k_{y} \Lambda_{0} \simeq k_{y} /\left(1+k_{\perp}^{2} \rho_{c}^{2}\right) \text {. }
$$

The corresponding transport is given by the expression for the particle flux $\Gamma_{s}=\sum_{k}\left(c k_{y} / B\right) J_{k}\left(e_{s} n_{0 s} / T_{s}\right) / m\left(g_{s}\right)$, with a similar expression for the heat flux. Here, $I_{k} \equiv\left\langle\left|\phi_{k}\right|^{2}\right\rangle$ is the intensity of the turbulent spectrum. Using $g_{i}$ in Eq. (2), this gives, for the ions, $\Gamma_{i}=-D_{i} n_{0 i}^{\prime}$, where

$$
D_{i}=\sum_{\mathbf{k}}\left(c k_{y} / B\right)^{2} I_{\mathbf{k}} \Lambda_{0} \gamma / \omega_{r}^{2}
$$

From Eq. (3), the factor $\Lambda_{0} \gamma / w_{\tau}^{2}$ here equals $\alpha / \omega_{\text {eee, }}$ and as a result orbitaveraging does not enter the shown expression for $D_{i}$ explicitly, but only through its modification of $I_{k}$, and perhaps of $\alpha$.

Obtaining $I_{\mathbf{k}}$ correctly through a full nonlinear calculation is difficult, and is not attempted here. The usual shortcut is to invoke the 'mixinglength prescription' $(e / T)^{2} \sum_{k} I_{k} \simeq 1 /\left(\Delta k_{\perp} L_{n}\right)^{2}$, which applied to Eq. (4) for the conventional $\tau \geq 1$ theory, for which $\Delta k_{\perp} \simeq \rho_{s}^{-1}$, yjelds $D \simeq$ $\gamma / \Delta k_{\perp}^{2} \simeq \alpha D_{0}$, where $D_{0} \equiv \rho_{s}^{2} c_{s} / L_{n}$ is the 'gyro-reduced Bohm' expression, scaling as $T_{e}^{3 / 2}$. Including nonzero $b_{b}$ in the $\tau \gtrsim 1$ theory, for which $\Delta k_{\perp} \simeq$ $p_{t}^{-1}$, results in $D \simeq \alpha D_{0}\left(\rho_{t} / \rho_{s}\right)$. The factor $\left(\rho_{t} / \rho_{s}\right)=\left(\rho_{c} / \rho_{g i}\right) \simeq\left(1+q^{2}\right)^{1 / 2}$ introduced in going from the slab theory to its toroidal modification is reminiscent of a similar factor introduced in going from classical to neoclassical 
transport, and introduces an additional dependence $\simeq q \propto B / I_{p}$ into $D_{i}$ or $\chi_{i}$. As is well known, the scaling $D, \chi \propto T_{e}^{3 / 2} / B^{2}$ characteristic - $f$ conventional drift-wave theories (of which the $b_{b}=0, \tau \geqslant 1$ TEM theory just discussed is an example) conflicts with the experimentally observed scaling with $I_{p}$ and $B$, and must be supplemented by a factor of roughly $q^{2.5}$ to be brought into agreement. ${ }^{19}$ The additional factor of $q$ which orbit averaging introduces into the $\tau \gtrsim 1$ result thus partially remedies one of the major failings to date of drift wave theories. A similar modifying factor has been found by Guo, et al. ${ }^{20}$ for the ITG mode in going from slat to toroidal geometry, due not to the drift-averaging considered here (which is not present, due to the more standard frequency ordering made there), but to toroidal effects in the ballooning equation, which are not considered here.

To consider the modification of this result in the $T<1$ regime, we assume that the saturated amplitude of $I_{\mathbf{k}}$ is proportional to a positive power $p$ of $\gamma$, reflecting the intuition that the saturation amplitudes should become snaller as the term driving them diminishes. While the power implied by the simplest mixing-length argument just noted is $p=0$, contradicting this intuition, weak turbulence calculations suggest that $p$ should instead equal unity ${ }^{17}$, while scaling arguments for strong turbulence suggest ${ }^{18} p=2$. Here we take $p=1$. Then one has

$$
\frac{D(\tau<1)}{D(\tau>1)} \simeq \frac{\int d k k_{y} /\left(1+k_{\perp}^{2} \rho_{c}^{2}\right)}{\int d k k_{y} /\left(1+k_{\perp}^{2} \rho_{l}^{2}\right)^{2}} \simeq \frac{\rho_{l}^{2 r}}{\rho_{c}^{2 r}}=\tau^{r},
$$

where the exponent $r$, lying between 1 and 2 , depends upon the scading one assumes for the spectral widths in $k_{k}$ and $k_{r}$. The scaling from the simple analytic theory $\left(k_{\|} \sim k_{\mathrm{v}}, k_{\mathrm{r}} \sim k_{y}^{0}\right)$ gives $r=3 / 2$. Thus, while the TEM scaling for L-mode discharges is $D(t>1) \propto T_{e}^{3 / 2}$, for supershots, 
one expects $D(\tau<1) \propto T_{e}^{3 / 2} \tau^{r}$. Therefore, if $T_{\mathrm{r}}$ increases only weakly as $T_{i}$ increases, as is the case for the supershot data in Fig.1, one expects the inverse scaling of $D_{i}$ or $\chi_{i}$ with $T_{i}$.

For the ITG mode, one may neglect $\alpha$ in Eq. (2), but now finite $k_{\|}$is essential, and must be determined by converting the local dispersion relation (2) into the appropriate nonlocal mode equation, as in Ref. 8. As discussed in Ref. 8, the largest growth rate occurs when the second term in Eq. (2) about cancels the term in $K_{3}$, hence for $k_{\perp}$ satisfying $1 \simeq \tau\left(1-\Lambda_{0}\right) K_{3}$, reducing to $1 \simeq k_{\perp}^{2} \rho_{a}^{2} K$ in the usual slab treatment, or $1 \simeq k_{1}^{2} \rho_{t}^{2} K$ for $b_{b} \neq 0$. Thus, the growth rate $\gamma$ peaks around $k_{\perp} \rho_{l} \simeq K^{-1 / 2}$ (sketched in Fig.2), with value given by

$$
\Omega \equiv \omega / \omega_{\cdot e} \simeq\left(\frac{\rho_{l}}{\rho_{s}}\right)^{1 / 2}\left(\frac{\Lambda_{0}^{\prime}}{\Lambda_{0}}\right)^{1 / 4}\left[\frac{\Lambda_{0} K_{4} \bar{s}}{\left[1+\tau\left(1-\Lambda_{0}\right)\right]}\right]^{1 / 2},
$$

with $\tilde{s} \equiv(2 l+1) s$, and $l$ the radial mode number, where for the fastest growing mode $l$ decreases with increasing $s$ so as to keep $\dot{s}$ about constant.

Since $\Delta k_{\perp} \simeq K^{-1 / 2} \rho_{t}^{-1}=\left(1+\eta_{i}\right)^{-1 / 2} \rho_{c}^{-1}$ is small, one may take $\Lambda_{0} \simeq$ $1, K_{4} \simeq K$ in $\Omega$. And since it depends only on $T_{i}$ for all values of $T$, the transition from a large- $\tau$ to a small- $\tau$ regime evidenced by the TEM mode does not occur for the ITG mode. Using the given expressions for $\Omega$ or $\gamma$ and $\Delta k_{1}$ for the single ITG regime, we summarize the overall discussion, by displaying the expressions for $\chi_{i}$ including the orbit-averaging effects, for the two TEM regimes, and the single ITG regime, followed by the approximate scalings of these results with $T_{e}, T_{i} B$, and $I_{p}$ :

$$
\begin{aligned}
\chi_{i}(T E M, \tau \geqslant 1) & \simeq\left(\rho_{t} / \rho_{s}\right) \alpha D_{0} \sim T_{e}^{3 / 2} B^{-1} I_{p}^{-1} \alpha \\
\chi_{i}(T E M, \tau<1) & \simeq\left(\rho_{t} / \rho_{s}\right) \alpha \tau^{r} D_{0} \sim T_{e}^{3 / 2++} T_{i}^{-*} B^{-1} I_{p}^{-1} \alpha \\
\chi_{i}(I T G) & \simeq\left(\rho_{t} / \rho_{s}\right)^{3 / 2} K^{-1 / 2} D_{0} \sim T_{e}^{1 / 2} T_{i} B^{-1 / 2} J_{p}^{-3 / 2} .
\end{aligned}
$$


For all three results, one notes that the 'neociassical factor' $\left(\rho_{t} / p_{s}\right) \simeq$ $\left(1+q^{2}\right)^{1 / 2} \sim q$ improves the agreement with th experimental scaling ${ }^{19}$ $\chi_{\text {exp }} \sim T^{3 / 2} B^{-2} q^{5 / 2}$ over the scaing $\lambda\left(b_{b}=0\right) \sim \Gamma^{3 / 2} B^{-2}$ characteristic of earlier drift-wave theories, with the ITG mode showing the strongest improvement. The factor is a gentric aspect of the theory, not specific to the particular modes considered here. As already noted, the twn TEM regimes display the scaling with $\tau$ jeeded to explain the difference between L-mode and supershots exhibjted in Fig.1, while the single ITG scaling does not. $A$ preliminary TFTR database test of the expected TEM scaling for $r<1$, made using the supershot discharges of Fig.J, yields a $T_{i}$ dependence $\chi_{i} \sim$ $T_{i}^{-r}$, with $r \simeq 1.44$. However, the remaining $T_{e}$ tependence appears weaker than the $T_{\mathrm{e}}^{3 i^{2+1}}$ ii $\Sigma_{q}$. (7), suggesting that the dejendence $D_{0} \sim T_{\mathrm{e}}^{3 / 2}$ from the usual TEM theory should be - eaker, or that transport is controlled by some other mode with 2 two $\tau$-regime oehavjor similar to that manifested by the TEM here, but with weaker $T_{c}$ sca'ing.

We rave inclided in the TFM scalings the factor $\alpha$. The scaling of this factor remains to be more carefully explored, but further refinements of existing TEM theory have been suggested ${ }^{19}$ which might, along with the ballooning effect poinied out in Ref. 20 notcd above, introduce she needed remaining $q$ dependtence into a more complete theory.

These result 3 suggest a reinterpretation of the cause $s$ the geod confinement and the very high ion temperatures in TFTR supershots, which had been previously attributed to the stabilization of ITG due to peaked density profiles. ${ }^{21}$ The present result implies the possibility that the supershot ceritral ioli temperatures 'run away,' (at least to the equipartition limit of tho average beam energy) due to the $\chi_{i} \propto T_{i}^{-r}$ scaling of the orbit-averaged 
TEM transport for $\tau<1$, and perhaps also that the peaked density profles are caused by the high- $T_{i}$ orbit-averaging effect on $D$. These conjectures still need to be further tested against the experimental databasea, and against numerical solutions containing the orbit-avesaging effect. ${ }^{16}$

\section{Acknowledgments}

The authors are grateful to T.S. Hahm, J.A. Krommes, G. Rewoldt, S.D. Scot:, and W.M. Tang for informative discussions.

This work supported by U.S.Department of Energy Contract No.DEAC02-76-CHO3073. 


\section{References}

${ }^{1}$ H.E.Mynick,J.A. Krommes, Phys.Rev.Lett. 43, 1506-1509 (1979).

${ }^{2}$ H.E.Mynick,J.A. Krommes, Phys.Fluids 23, 1229-1237 (1980).

${ }^{3}$ H.E.Mynick,J.D. Strachan, Phys.Fluids 24, 695 (1981).

${ }^{4}$ R.H. Radetsky, S.D. Scott, R. Kaita, R.J. Goldston, G.W. Hammett, et al., Proc. 15th European Conference on Controlled Fusion and Plasma Physics, Dubrovaik, 1988, 79, S.Pesic and J. Jacquinot (eds.), (European Physical Saciety, 1988).

${ }^{5}$ S.J. Zweben, R.E. Duvall, E.D. Fredrickson, R.J. Goldston, H.E. Mynick, J.D. Strachan, and R.B. White,Phys. Fluids B2, 141 ( (1990).

${ }^{6}$ R.E. Duvall, Ph.D. Dissertation, Princeton University (1990).

־C.S. Liu, M.N. RosenbJuth, W.M. Tang, Phys.Fluids 101040 (1976).

${ }^{8}$ S. Hamaguchi, W. Horton, Phys.Fluids B2, 1833 (1990).

9S.D. Scott, C.W. Barnes, L.R. Grisham, G. Hammett, W.W. Heidbrink, et al., Proceedings of the 13 th International Conference on Plasma Physics and Controlled Nuclear Fusion Research (Washington, DC, October, 1990) (International Atomic Energy Agency, Vienna, Austria), Paper IAEA-CN-53/A-3-6 (1990).

${ }^{10}$ P.C. Efthimion, Phys.Fluids B (1991) (to be published).

${ }^{11}$ A. N. Kaufman, Phys. Fiujis 15, 1063 (1972). 
${ }^{12}$ R. D. Hazeltine, S. M. Mahajan and D. A. Hitchcock, Phys. Fluids 24, $1164(1981)$.

${ }^{13}$ I.B. Bernstein and K. Molvig, Phys. Fluids 26, 1488 (1983).

${ }^{14}$ H. E. Mynick and R. E. Duvall, Phys.Fluids-B 1, 750 ;1989).

${ }^{15} \mathrm{G}$. Rewoldt, private communications (1090).

${ }^{16}$ G. Rewoldt, W.M. Tang, Phys. Fluids B2, 318 (1990).

17T.S. Hahm, W.M. Tang, Phys. Fluids B2, 1815 (1990).

${ }^{18}$ \.A. Krommes, private communications (1990).

I9W.M. Tang, Princeton University Report PPPL-2728 (November, 1990).

${ }^{20}$ S.C. Guo, L. Chen, S.T. Tsaj, P.N. Guzdar, Plasma Physics and Controlled Fusion 31423 (1989).

${ }^{21}$ S.D. Scott, P.H. Diamond, R. Fonck, R.J. Goldston, B. Howell, et al, Phys. Rev. Letters 64531 (1990). 


\section{Figure Captions}

Figure 1. Experimental measurements of $\chi_{i}$ versus $T_{i}$ on TFTR, for L-mode and supershot discharges.

Figure 2. Sketch of growth rate - us $k_{\perp} \rho_{s}$, for the single-t regime oi 'mode, for the usual lEill theory $\left(\tau \geq 1, b_{b}=0\right.$ ), and for the moa: of that result by orbit averaging, in the $\tau \geq 1$ and $\tau<1$ regimes. 


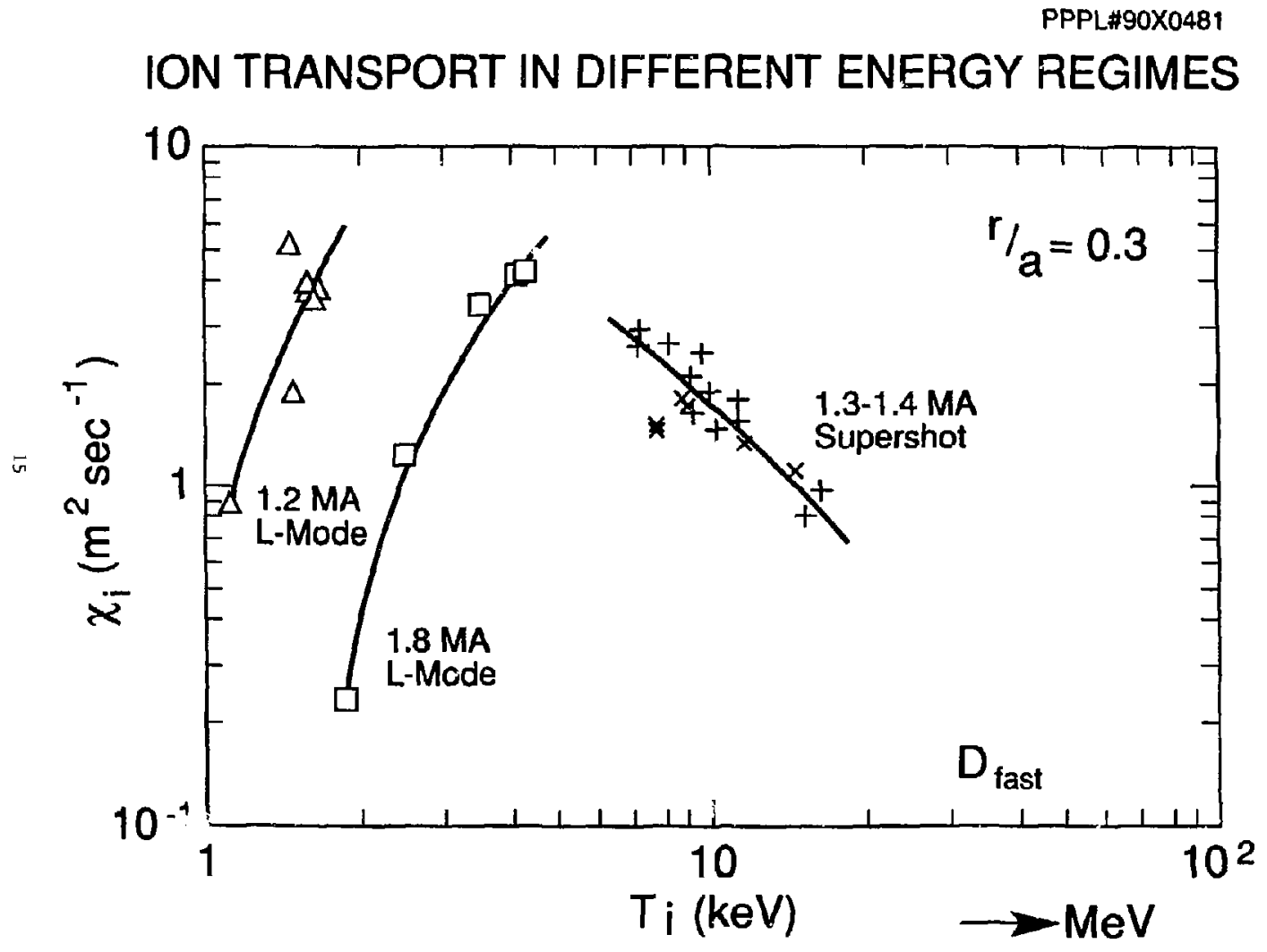

Fig. I 


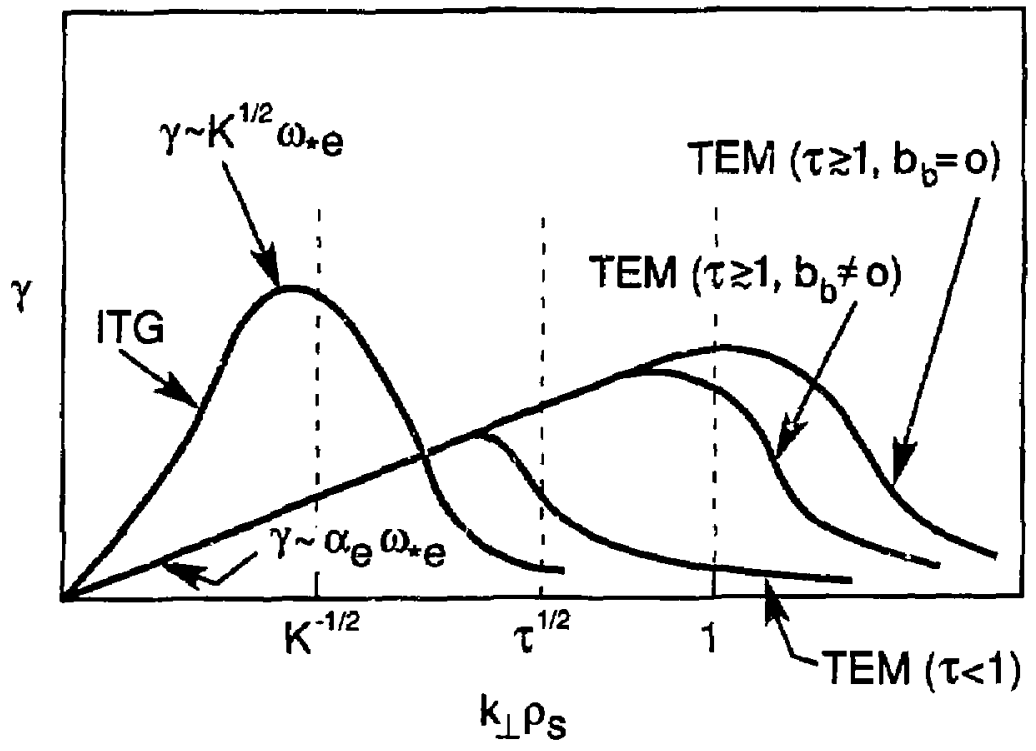

Fig. 2 